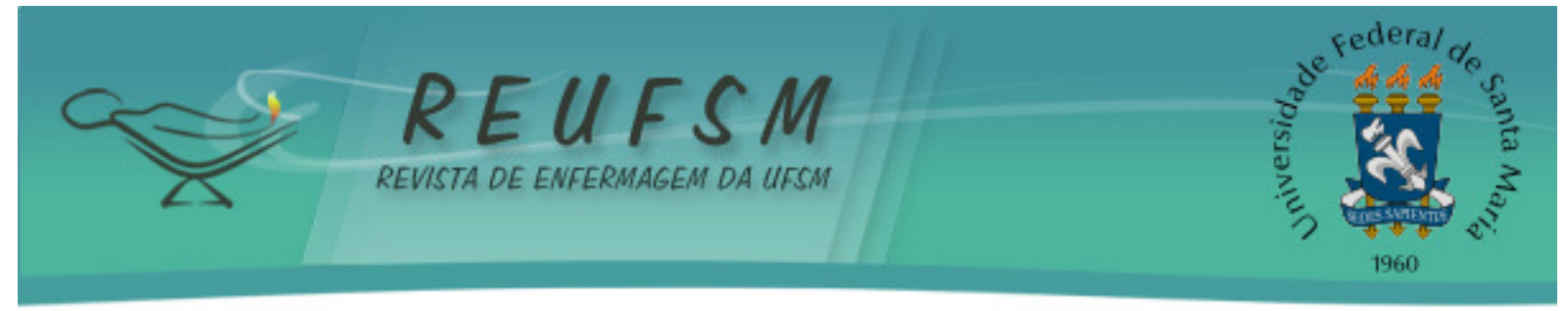

RELATO DE EXPERIÊNCIA

\title{
COLÔMBIA: CENÁRIO DE ESTUDO EM ENFERMAGEM NO PROGRAMA DE MOBILIDADE ACADÊMICA INTERNACIONAL
}

\section{COLOMBIA: A STUDY SCENARIO IN NURSING IN THE PROGRAM OF INTERNATIONAL ACADEMIC MOBILITY}

\section{COLOMBIA: ESTUDIO DEL ESCENARIO DE ENFERMERÍA EN EL PROGRAMA DE MOVILIDAD ACADÉMICA INTERNACIONAL}

Doi: $10.5902 / 2179769218952$

Bianca Contreira de Jung ${ }^{1}$

Vera Lucia Freitag ${ }^{2}$

Roxana Isabel Cardozo Gonzáles ${ }^{3}$ Indiara Sartori Dalmolin ${ }^{4}$

RESUMO: Objetivo: relatar a experiência de uma estudante de enfermagem em Programa de Mobilidade Acadêmica Internacional na Colômbia. Metodologia: relato de experiência crítico e reflexivo realizado na Colômbia durante o primeiro semestre de 2012. Resultados: a experiência oportunizou vivenciar atividades teóricas e práticas, participação na organização de eventos científicos, apresentação de trabalhos acadêmicos em congressos e aproximação ao campo de pesquisa no cenário colombiano, com a garantia de atuar em um cotidiano de estudos específicos e singulares no âmbito da enfermagem. Considerações Finais: como contribuições deseja-se estimular os estudantes para que façam mobilidade na graduação, intensificando o aperfeiçoamento acadêmico e profissional e, assim, contribuindo para o ensino superior de qualidade e aberto à integração dos países.

Descritores: Intercâmbio educacional internacional; Educação; Estudantes de enfermagem; Aprendizagem.

ABSTRACT: Aim: to report the experience of a nursing student in International Academic Mobility Program in Colombia. Methodology: critical and reflective report of the experience held in Colombia during the first semester of 2012. Results: the experience made possible to experience theoretical and practical activities, participation in the organization of scientific events, presentation of academic papers at conferences and access to the research field in the Colombian scenario, with the guaranty of working on an everyday specific and singular studies in nursing. Final Remarks: as contributions, there is a will to stimulate the students to perform mobility in their graduation, intensifying the academic and professional improvement and thus contributing to quality higher education and opening it to the integration between countries.

Descriptors: International educational exchange; Education; Nursing students; Learning.

\footnotetext{
${ }^{1}$ Enfermeira. Mestre em Ciências pela Universidade Federal de Pelotas (UFPel). Especialista em Gestão de Organização Pública em Saúde pela Universidade de Santa Maria (UFSM). Pelotas, RS, Brasil. E-mail: biajung@bol.com.br

2 Enfermeira. Mestre em Ciências pela Universidade Federal de Pelotas (UFPel). Especialista em Gestão de Organização Pública em Saúdepela Universidade de Santa Maria (UFSM). Pelotas, RS, Brasil. E-mail: verafreitag@hotmail.com

${ }^{3}$ Enfermeira. Pós-Doutora em Enfermagem Saúde Pública pela Escola de Enfermagem de Ribeirão Preto da Universidade de São Paulo (EERP/USP). Professora Doutora na Faculdade de Enfermagem da Universidade Federal de Pelotas. Pelotas, RS, Brasil. E-mail: roxana_cardozo@hotmail.com

${ }^{4}$ Enfermeira. Residência Multiprofissional em Saúde da Família, Universidade Federal de Santa Catarina. Email: indi2007dalmolin@hotmail.com
} 


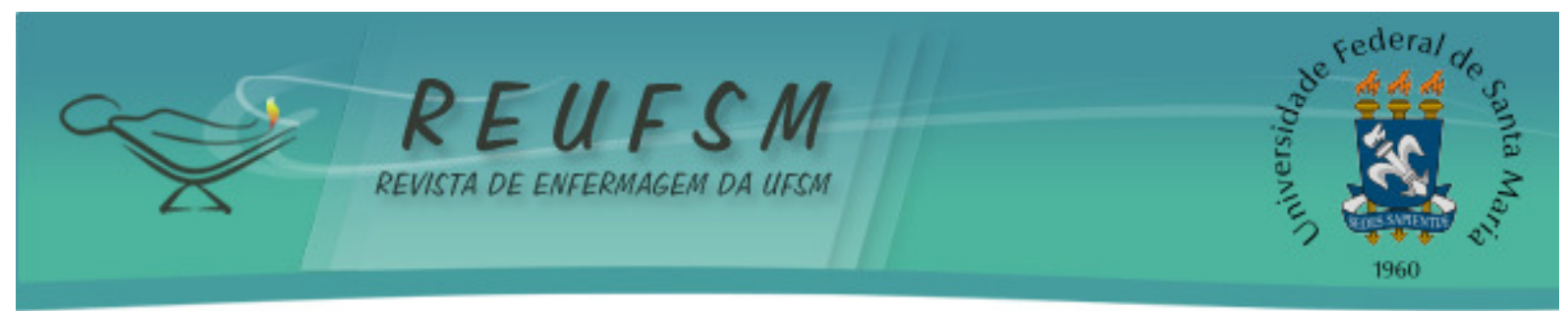

RESUMEN: Objetivo: presentar la experiencia de una estudiante de enfermería en el Programa de Movilidad Académica Internacional en Colombia. Metodología: informe de la experiencia crítica y reflexiva en Colombia en el primer semestre de 2012. Resultados: la experiencia propició la experiencia en actividades teóricas y prácticas, la participación en la organización de eventos científicos, presentación de trabajos académicos en conferencias y el acercamiento al campo de investigación en el escenario colombiano, con la garantía de actuar en un cotidiano de estudios específicos y únicos en enfermería. Notas Finales: hay el intuito de estimular los estudiantes para que hagan movilidad en la graduación, intensificando la mejora académica y profesional y, con esto, contribuyendo para una educación superior de calidad y abierta a la integración entre los países.

Descriptores: Intercambio educacional internacional; Educación; Estudiantes de enfermería; Aprendizaje.

\section{INTRODUÇÃO}

Os programas de intercâmbio buscam promover o câmbio entre os sistemas educacionais de outros países em relação ao conhecimento, à cultura, a tecnologia e a inovação. Assim, esta oportunidade torna-se valiosa para os estudantes, pois permeia conhecer novas culturas, sistemas políticos e organizações sociais, aprimorar e ter contato com outros idiomas, além de incluir a bagagem da aquisição de conhecimentos.

A experiência de viver em outro país proporciona conhecer hábitos diferentes e peculiares, novas perspectivas de ensino e aprendizagem, auxiliar na superação de dificuldades, pois o estudante precisa se adaptar ao ambiente, enfrentar desafios e crescer no âmbito pessoal e profissional. ${ }^{1-13}$

Nessa conjuntura, a mobilidade apresenta-se como um meio de alcançar um diferencial para a formação profissional, visando o aprendizado significativo, o qual repercute diretamente na formação crítica e reflexiva dos estudantes. Essa formação assume um caráter baseado em responsabilidades que os estudantes precisam ter para a sua adaptação em diferentes contextos. ${ }^{2}$

Reconhece-se a importância de estudantes e futuros profissionais engajados na luta por uma sociedade plural, que valorize o ser humano em sua interface cultural, étnica, social e relacional. ${ }^{3}$ Este contexto envolve o interesse por outros idiomas, além da língua materna e a cultura de outros países e povos, sendo que o estar em países diferentes contribui, expressivamente, no processo de formação do aluno, capacitando-o para lidar com adversidades e necessidades encontradas na trajetória pessoal e profissional. ${ }^{4}$

Para tal, é necessário, também, a integração do ensino à pesquisa, bem como o estabelecimento de uma relação interdependente e construtiva, aproximando o sítio pedagógico ao científico. ${ }^{5}$ Além disso, favorece a formação do enfermeiro com perfil crítico e reflexivo, com competências baseadas na tomada de decisões, comunicação, liderança, administração e gerenciamento e educação permanente. ${ }^{6}$

Assim, apreender o sistema educativo na área da enfermagem em uma realidade distinta ao Brasil é uma oportunidade valiosa para conhecer o ensino e processo de trabalho dos profissionais. ${ }^{7}$ Considera-se que a forma como a profissão opera em relação ao cuidado permitirá a identificação de potencialidades e fragilidades relacionadas ao processo de cuidar.

Justifica-se que o interesse em publicar este relato de experiência deve-se ao fato de poder compartilhar o conhecimento produzido com a comunidade acadêmica, repercutindo na formação de estudantes e incentivando-os sobre a importância para o desenvolvimento pessoal e profissional. Além de poder valorizar a realidade de um país, 


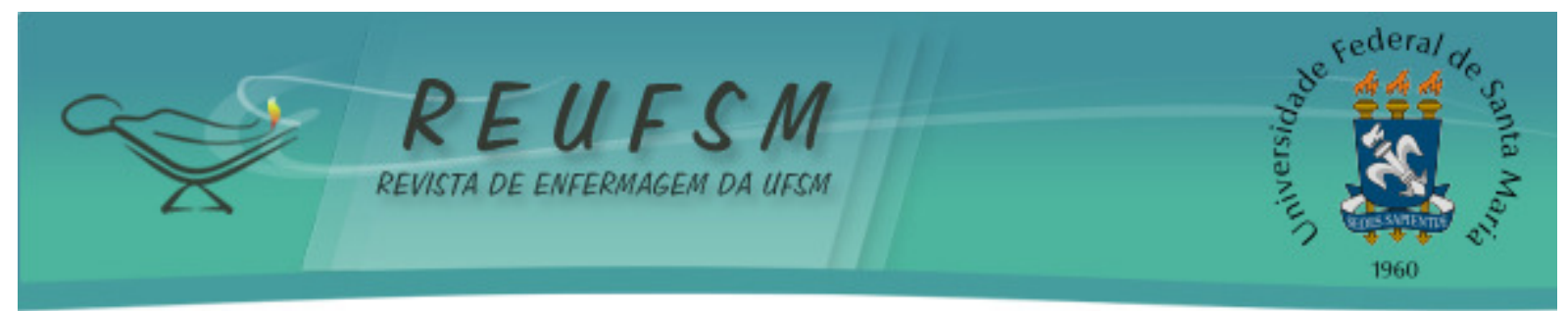

tanto social como culturalmente, do ponto de vista de sua receptividade aos estudantes estrangeiros e na disponibilidade de universidades com ensino de qualidade.

Em razão da sua relevância na formação acadêmica, o presente trabalho tem como objetivo relatar a experiência de uma estudante de enfermagem em Programa de Mobilidade Acadêmica Internacional na Colômbia, a partir de suas vivências, observações e atividades realizadas no cenário de atuação.

\section{MÉTODO}

Relato de experiência de cunho crítico e reflexivo de uma estudante no Programa de Mobilidade Acadêmica Internacional da Universidade Federal de Pelotas (UFPel), durante o primeiro semestre de 2012, na Colômbia.

A mobilidade acadêmica no âmbito internacional tem por objetivo criar condições para que estudantes das Universidades tenham oportunidades de convívio e de aprendizado em novo ambiente acadêmico, linguístico e cultural, contribuindo com sua formação intelectual, profissional e humana, bem como com o desenvolvimento de sua competência intercultural. ${ }^{8}$

0 relato descreve etapas vivenciadas a partir de como o intercâmbio começou a ser pensando, decisão pela universidade na Colômbia, trajetória de atividades na mobilidade acadêmica, importância do intercâmbio para a formação dos estudantes de enfermagem, cenário de formação acadêmica em enfermagem da Universidad de Ciencias Aplicadas y Ambientales e o olhar para o sistema de saúde colombiano.

A Universidad de Ciencias Aplicadas y Ambientales (UDCA) foi o cenário de desenvolvimento das atividades teóricas, localizada em Bogotá/Colômbia. Além do cenário teórico, os estágios ocorreram nos seguintes espaços: Hospital San Rafael De Facatativá, na cidade de Facatativá/Colômbia, San Blass e Soleec Enfermeras Clinica Santafe em Bogotá.

A escolha dos campos teóricos e práticos aconteceu por meio de um plano de estudos, elaborado em conjunto pela orientadora brasileira e colombiana, com o aval do departamento de enfermagem da universidade estrangeira. Este plano permitiu o maior aproveitamento da experiência, a organização das atividades diárias e a otimização do tempo, a fim de direcionar os pontos de atenção e interesse da estudante.

Foram utilizados alguns critérios para a escolha do destino da mobilidade acadêmica, sendo eles a empatia pelo país, o gosto pelo idioma espanhol, a alta incidência de tuberculose, temática de estudo e pesquisas da autora principal e pela América Latina possuir diversidade de tipos de instituições de educação superior.Fato este que estimulou a discente para o intercâmbio em um país latino americano, de modo a incentivar aos demais estudantes a reconhecer o ensino de qualidade que os países do sul da América oferecem.

\section{RESULTADOS E DISCUSSÃO}

\section{Relato da experiência do Programa de Mobilidade Acadêmica Internacional}

A decisão pela mobilidade ocorreu ao cursar $08^{\circ}$ semestre do Curso de Enfermagem da UFPel e se deu pela necessidade particular de aprimorar os conhecimentos em enfermagem. Além disso, o desejo de conhecer e vivenciar culturas diferentes e compreender a conformação dos aspectos culturais no processo de cuidar, o interesse pelo aprendizado do idioma espanhol e a possibilidade de criar vínculos científicos para favorecer a troca de experiências. 


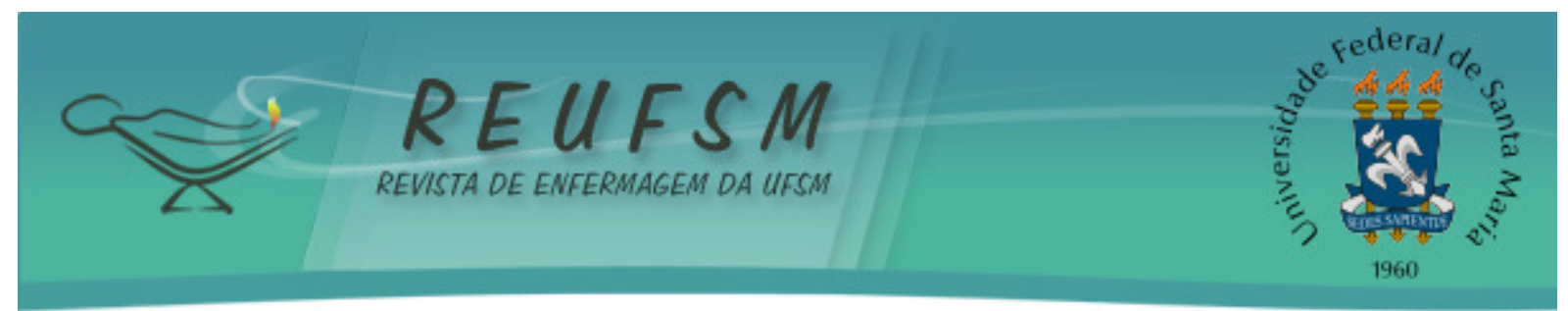

Neste período, a estudante esteve inserida em projeto de pesquisa desenvolvendo estudos sobre a avaliação dos serviços de saúde para a atenção à tuberculose. A partir deste contexto, foi possível ampliar o olhar para o "mundo da pesquisa" e perceber a importância do desenvolvimento de pesquisas na área da enfermagem para além do âmbito nacional, aumentando o saber relacionado à doença e as formas de cuidado.

A interface pesquisa no exterior colabora para promover a consolidação, expansão e internacionalização da ciência, tecnologia e inovação, por meio da mobilidade internacional. Pesquisar no exterior favorece a construção do conhecimento entre estudantes de diferentes países, com o propósito de gerir mudanças nas práticas de saúde, permeadas por desafios, com a necessidade de superar barreiras não apenas geográficas, mas culturais, de idioma e contexto social, econômico e político.

A opção pela Universidad de Ciencias Aplicadas y Ambientales ocorreu devido à excelência acadêmica que o curso de Enfermagem possui, programa acreditado de alta qualidadesob o número13483 de 23 de outubro de 2012 pelo Ministério da Educação Nacional. ${ }^{9}$ Valoriza o exercício da enfermagem e dos aspectos do processo saúde e doença de forma singular e holística, com uma visão global das necessidades que envolvem o cuidar.

\section{Trajetória de Atividades na Mobilidade Acadêmica}

A trajetória de experiências proporcionadas pela mobilidade acadêmica permeou momentos de vivências teóricas e práticas em enfermagem no contexto da Colômbia, possibilitando compartilhar e integrar os grupos de estudos com colegas; trocar experiências da realidade local em pesquisas e participar da organização de eventos e exposição de trabalhos em congressos científicos.

Em destaque, a experiência da enfermagem colombiana possibilitou conhecer semelhanças e diferenças desta área da saúde, revelando convergências, similaridades e grandes diferenças na organização e distribuição dos programas e políticas de saúde.

Elegeu-se duas disciplinas correspondentes ao último semestre dos alunos da UDCA: Cuidado deEnfermería en Programas Especiales e Investigación en Enfermería. Metodologicamente as disciplinas apresentaram-se de maneira distinta, utilizando de recursos variados para facilitar o processo de ensino e aprendizagem, que perpassaram atividades interdisciplinares e interinstitucionais.

O componente curricular "Cuidado deEnfermería en Programas Especiales" apresentou uma metodologia mais complexa por envolver aspectos de componente teórico e prático. $\mathrm{O}$ que enalteceu essa disciplina quando comparada às disciplinas similares na universidade de origem (Cuidado do Adulto e Enfermagem em Urgência e Emergência), foi a realização de uma abordagem teórica e prática para preparar os estudantes em situações particulares que poderiam enfrentar na vida laboral. Esses momentos envolveram cursos de suporte básico de vida e avançado e exames diagnósticos como eletrocardiograma.

O componente prático possibilitou a inserção da estudante no sistema de saúde da Colômbia, aproximando-se de usuários/pacientes, profissionais e colegas, permitindo a apreciação da dinâmica do trabalho da enfermagem no contexto local.

Segundo o plano de estudos pactuado entre a estudante e a coordenação de enfermagem, definiu-se a sua participação nos seguintes campos práticos: Unidade de Terapia Intensiva Neonatal, Unidade de Urgência e Emergência, Unidade Clínica e Cirúrgica e na Hospitalização Domiciliar.

As atividades em Terapia Intensiva Neonatal e hospitalização domiciliar não seriam contempladas no currículo do curso de enfermagem de origem. Uma experiência 


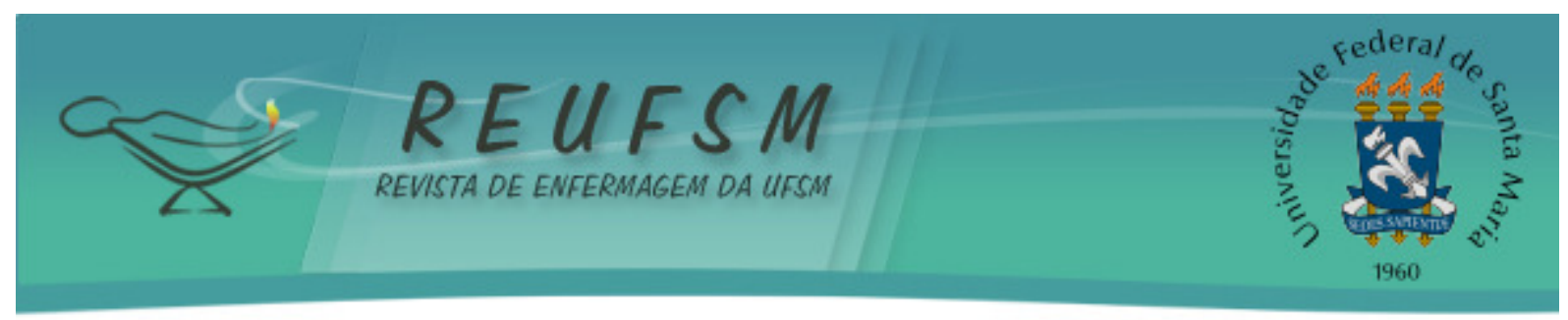

semelhante aponta que os estágios realizados no exterior impulsionam um paralelo com a realidade local, instigando possíveis replicações de fortalezas. ${ }^{10}$

Estudo ${ }^{11}$ sobre o relato de experiência em atividades desenvolvidas com disciplinas cursadas no exterior mencionou a importância destas disciplinas para o aprimoramento técnico-científico e a troca de experiência com os colegas de classe. Além de ser um momento rico para a construção de discussões em sala de aula.

No que tange a disciplina "Investigación en Enfermería", iniciaram-se as atividades de pesquisa direcionadas para o panorama mundial da pesquisa em enfermagem. A participação no componente resultou na produção científica do seguinte trabalho: "Panorama Epidemiologico de la Tuberculosis en Colombia en el periodo de 2009-2012".

Este estudo foi produzido em parceria com os colegas, de modo que aconteceram encontros para problematizar a realidade da tuberculose na Colômbia e para socializar o panorama da doença no Brasil. Nesse contexto, foram identificadas fragilidades comuns às encontradas no Brasil e potencialidades que são possíveis de reprodução, despertando o interesse de solucionar os impasses detectados.

Estudo aponta a importância do estabelecimento de parcerias com pesquisadores estrangeiros em busca de uma maior cooperação científica. ${ }^{12-18}$ Dessa forma, a pesquisa no exterior contribui para que instituições brasileiras e estrangeiras possam se conectar com a finalidade de produzir ciência e tecnologia, compartilhar a inovação científica e partilhar de suas distintas realidades entre países.

Destaca-se a participação em atividades extracurriculares, na organização do evento Primer Encuentro Internacional de Enfermería en Atención Domiciliaria, reunindo pesquisadores de países das Américas, como Estados Unidos, Brasil, Uruguai, México e Costa Rica. Durante este evento, apresentou-se um pôster com o seguinte tema: Las prácticas en atención domiciliaria en la Universidad Federal de Pelotas en Brasil. Momento este, de crescimento acadêmico para a estudante, pois possibilitou a troca de experiências com diversos pesquisadores e o aprimoramento do idioma.

Corroborando com essa discussão, autores afirmam que aproveitar o intercâmbio para socializar os estudos desenvolvidos na instituição de origem, faz da experiência uma oportunidade singular para compartilhar a cultura, os problemas, as políticas e as ações desenvolvidas na perspectiva do trinômio: universidade, comunidade e serviços de saúde no Brasil. ${ }^{13}$

\section{Importância do Intercâmbio para a Formação dos Estudantes de Enfermagem}

A experiência de intercâmbio tornou-se importante para a formação dos estudantes de enfermagem, pois influenciou no atendimento holístico e baseado nas singularidades das pessoas, de modo a prestar uma assistência compatível com o mundo de especificidades no cotidiano das práticas de enfermagem. Dessa forma, os estudantes adquiriram maturidade para lidar com as situações complexas que envolvem o fazer saúde.

A realidade brasileira requer a expansão do processo de formação dos profissionais de enfermagem, passando a exigir experiências que sejam pautadas em diversos campos do conhecimento, incluindo os contextos culturais, sociais e os aspectos técnicos atinentes à profissão para gerar uma formação de enfermeiros críticos, reflexivos e agentes transformadores da realidade. ${ }^{14-17}$ Neste sentido, a importância da mobilidade torna-se premente, visto que a enfermagem necessita expandir seu potencial científico e consolidar-se enquanto campo da ciência, definindo suas particularidades naárea do conhecimento.

A participação em programas de mobilidade em enfermagem possibilita aos alunos maturidade pessoal e profissional, tornando esse momento rico e indispensável para a 


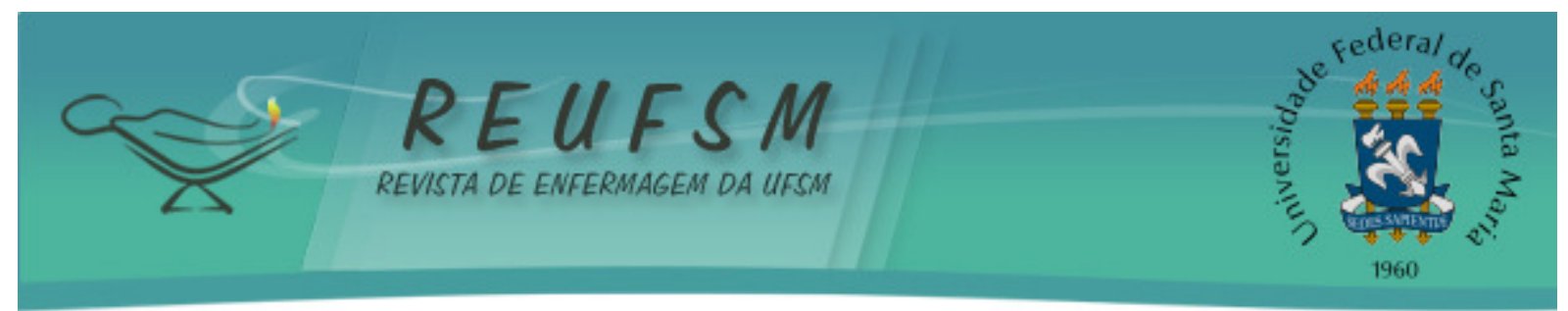

formação de laços acadêmicos. ${ }^{13}$ Os estudantes experimentam processos de trabalho em diferentes contextos e, com isso, entendem melhor o ato de cuidar.

\section{Cenário de Formação Acadêmica dos Futuros Enfermeiros na Realidade Colombiana}

O currículo de formação do curso de Enfermagem da UDCA reflete uma formação de compromisso com o desenvolvimento humano sustentável e situação de saúde do país. Os profissionais egressos podem atuar nos diversos cenários do setor saúde, com ênfase na resolução das necessidades em saúde do indivíduo, família e comunidade. ${ }^{9}$

O curso de enfermagem apresenta duração de nove semestres, com os primeiros quatro de disciplinas básicas e específicas de enfermagem. No quinto é desenvolvida a disciplina de saúde pública e nos seguintes as disciplinas de cuidado em enfermagem, estudo do sistema de saúde, saúde mental, e desenvolvimento do trabalho de conclusão de curso, respectivamente. ${ }^{9}$

Sobressai-se como diferencial no currículo em relação a realidade brasileira de ensino, a disciplina de inglês, cursada por quatro semestres e disciplinas de antropologia, pedagogia e didática em enfermagem estão presentes na grade curricular.

0 processo de construção e consolidação das profissões, em especial da enfermagem, acontece por aspectos que envolvem as condições socioculturais, históricas e políticas de determinado contexto, onde a profissão está situada. Assim, estes fatores produzem a identidade profissional, as condições e características atreladas à profissão, sendo que a enfermagem apresenta especificidades de acordo com a sua constituição.

A enfermagem colombiana a partir de 1919, ganha traços de uma profissão com bases científicas no ensino universitário. Assim, supera as raízes empíricas da formação vigente e passa a ter uma trajetória de ensino e aprendizagem em instituições assistenciais de saúde. ${ }^{15}$ Nesse sentido, o surgimento da Enfermagem e Escolas teve como âncora o modelo hospitalar de atenção individual e curativa, com a lógica de formação para o trabalho em hospitais. ${ }^{15}$

Dessa forma, a profissão na Colômbia surgiu com um perfil parecido ao da realidade brasileira, com influências e características semelhantes, porém com variações nos currículos das escolas, o que representa o aspecto singular de formação, de acordo com a instituição de ensino e com o sistema de saúde vigente.

\section{Tecendo o Olhar para o Sistema de Saúde Colombiano}

Atuar no sistema de saúde implicou na adaptação a uma realidade de ausência da universalidade, equidade e integralidade da atenção à saúde. Frente ao exposto, foi necessário conhecer o funcionamento do Sistema Geral de Seguridade Social em Saúde (SGSSS). ${ }^{16}$

Neste sistema, as pessoas pagam à empresa asseguradora que cobre os gastos em caso de adoecimento e se constituem afiliadas ao sistema. 0 mercado de saúde é dirigido por uma Empresa Promotora de Saúde (EPS), que atua como um intermediário financeiro e contrata a atenção de saúde de seus afiliados com as Instituições Prestadoras de Serviços de Saúde (IPS).

A diferença estrutural entre os sistemas de saúde brasileiro e colombiano reside nos seus princípios doutrinários e organizacionais. O Brasil tem o sistema de saúde com acesso universal a todos os serviços de saúdee a Colômbia tem o Plano Obrigatório de Saúde para todo o assegurado contributivo ou subsidiado, no qual o Governo assume a cobertura da assistência àqueles que não se encontram afiliados. 


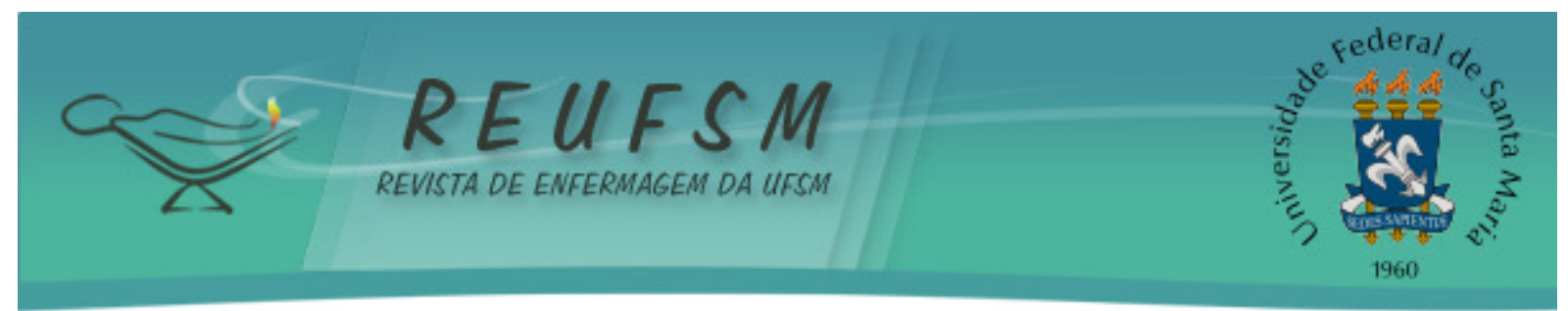

A aproximação com a realidade de saúde do país permitiu conhecer o real funcionamento do sistema e isso auxiliou na construção sobre a identidade profissional da enfermagem, observando-se que os enfermeiros assumem uma postura baseada, fortemente, no conhecimento clínico em suas práticas.

\section{CONSIDERAÇÕES FINAIS}

Destaca-se que a experiência do intercâmbio foi fundamental para a troca de informações e conhecimentos, para a valorização de uma nova cultura e idioma, aperfeiçoamento do conhecimento acadêmico e a formação de vínculo e laços de amizades. A importância de participação na mobilidade é algo que transpõe o desenvolvimento pessoal e acadêmico-profissional. Resulta em amadurecimento para a vida, fortalece o sentimento de benevolência e abertura a novas culturas.

Recomenda-se essa experiência aos acadêmicos que desejam obter uma formação singular e diferenciada, baseada em propostas de aprendizagens e na possibilidade de conhecer a atuação da enfermagem em países diferentes.

\section{REFERÊNCIAS}

1. Rodriguez Fernández N, Leal García E, Menendez Pérez M, Bermudez Alemán R, Perez Obregón BR, Fernandez López J. La internacionalización de la cultura: contribución a la formación integral de profesionales de la salud. Rev EDUMECENTRO. 2015;7(1):76-88.

2. Bubadué RM, Carnevale FCC, Paula CC, Padoin SMM, Neves ET. Participação em programa de intercâmbio internacional: contribuições da experiência de graduaçãosanduiche em enfermagem. Rev Enferm UFSM. 2013;3(3):555-62.

3. Castro AA, Cabral Neto A. O ensino superior: a mobilidade estudantil como estratégia de internacionalização na América Latina. Rev Lusófona de Educação. 2012;21(21):69-96.

4. Freire P. Pedagogia da autonomia: saberes necessários à prática educativa. São Paulo: Paz e Terra; 1996.

5. Massi L, Queiroz SL. Estudos sobre iniciação científica no Brasil: uma revisão. Cad Pesqui. 2010;40(139):173-97.

6. Corbellini VL, Santos BRLS, Ojeda BS, Gerhart LM, Eidt OR, Stein SC, et al. Nexos e desafios na formação profissional do enfermeiro. Rev Bras Enferm. 2010;63(4):555-60.

7. Mitre SM, Batista RS, Mendonça JMG, Pinto NMM, Meirelles CAB, Porto CP, et al. Metodologias ativas de ensino-aprendizagem na formação profissional em saúde: debates atuais. Ciênc Saúde Coletiva. 2008;13(2):2133-44.

8. Universidde Federal de Pelotas (UFPEL). Coordenação de Relações Internacionais. Pelotas; 2015 [acesso em 2015 out 27]. Disponível em: http://http://wp.ufpel.edu.br/crinter/.

9. Universidad de Ciencias Aplicadas y Ambientales [http://www.udca.edu.co]. Enfermería. [acesso em 2015 dez 17]. Disponível em: http://www.udca.edu.co/enfermeria/

10. Oliveira MG, Pagliuca LMF. Programa de Mobilidade Acadêmica internacional em enfermagem: relato de experiência. Rev Gaucha Enferm. 2012;33(1):195-8. 


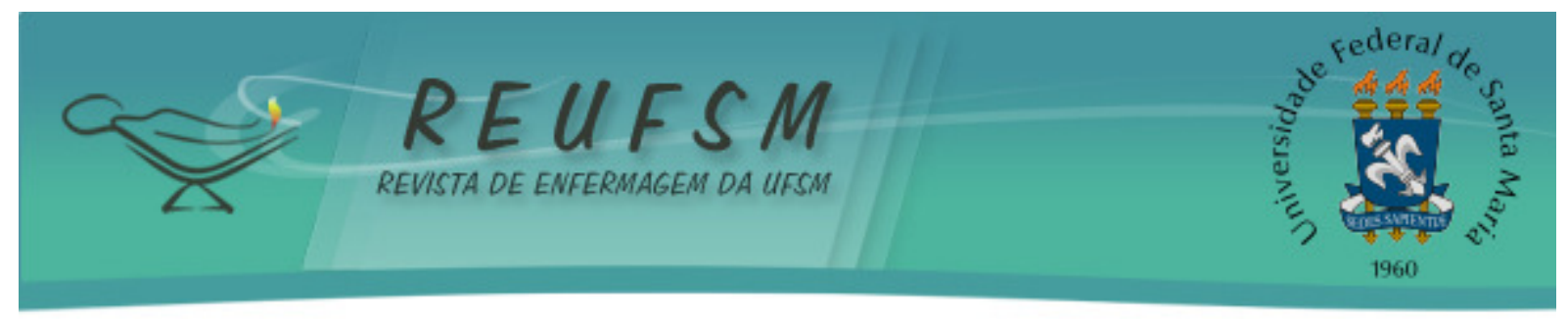

11. Mesquita MGR, Moreira MC, Malisk S. Em busca de conhecimento de enfermagem sobre o homem com câncer: uma experiência internacional. Esc Anna Nery Rev Enferm. 2009;13(2):421-24.

12. Blanco O, Marchitelli H, Varela E, Baccanelli M, Siufi G, Taura N. A internacionalización de la educación superior: como el instituto universitario gestiona esta nueva función estratégica. Rev Hosp Ital B Aires. 2012;(32)1:19-23.

13. Dalmolin IS, Pereira ER, Silva RMCRA, Gouveia MJB, Sardinheiro JJ. Intercâmbio acadêmico cultural internacional: uma experiência de crescimento pessoal e científico. Rev Bras Enferm. 2013;66(3):442-7.

14. Fernandes JD. Expansão de cursos/vagas de graduação em enfermagem e a qualidade do processo de formação da(o) enfermeira(o). Rev Bras Enferm. 2012;(65)3:395-6.

15. Carvajal BC. Los inicios de la profesionalización de la enfermería en Colombia. InvestEducEnferm. 2011;29(2):270-285.

16. Hernández M. Reforma sanitaria, equidad y derecho a la salud en Colombia. Cad Saúde Pública. 2002;18(4):991-1001.

17. Schomoeller R, Schveitzer MC, Arruda C, Backes VMS, Prado ML, Martini JG. Mercosul educativo na carreira de enfermagem. Rev Bras Enferm. 2012;65(5):856-61.

18. Salvetti MG, Bueno M, Gastaldo D, Kimura Af, Pimenta CAM. Doutorado sanduíche: considerações para uma experiência de sucesso no exterior. Rev Gaucha Enferm. 2013;(34):1:201-4.

Data de recebimento: 29/07/2015

Data de aceite: 04/12/2015

Contato do autor responsável: Bianca Contreira de Jung

Endereço postal: Rua Gomes Carneiro, $n^{\circ} 1$. Faculdade de Enfermagem da UFPel.

E-mail: biajung@bol.com.br 